\title{
Impact of needle-based confocal laser endomicroscopy on the therapeutic management of single pancreatic cystic lesions
}

\author{
Maxime Palazzo ${ }^{1}$ - Alain Sauvanet ${ }^{1} \cdot$ Rodica Gincul $^{2} \cdot$ Ivan Borbath $^{3} \cdot$ Goeffroy Vanbiervliet $^{4} \cdot$ Raphaël Bourdariat $^{2}$. \\ Anne-Isabelle Lemaistre ${ }^{5}$. Bertrand Pujol ${ }^{2}$. Fabrice Caillol ${ }^{6} \cdot$ Laurent Palazzo $^{7}$. Alain Aubert ${ }^{1}$. Frédérique Maire ${ }^{1}$. \\ Louis Buscail $^{8} \cdot$ Marc Giovannini $^{6} \cdot$ Sébastien Marque ${ }^{9} \cdot$ Bertrand Napoléon $^{2}$
}

Received: 5 May 2019 / Accepted: 5 August 2019 / Published online: 13 August 2019

(c) The Author(s) 2019

\begin{abstract}
Background and aim The diagnosis and therapeutic management of large single pancreatic cystic lesions (PCLs) represent major issues for clinicians and essentially rely on endoscopic ultrasound fine-needle aspiration (EUS-FNA) findings. Needlebased confocal laser endomicroscopy (nCLE) has high diagnostic performance for PCLs. This study aimed to evaluate the impact of nCLE on the therapeutic management of patients with single PCLs.

Methods Retrospective and comparative study. Five independent pancreatic disease experts from tertiary hospitals independently reviewed data from a prospective database of 206 patients with single PCL, larger than $2 \mathrm{~cm}$ and who underwent EUS-FNA and nCLE. Two evaluations were performed. The first one included the sequential review of clinical information, EUS report and FNA results. The second one included the same data + nCLE report. Participants had to propose a therapeutic management for each case.

Results The addition of nCLE to EUS-FNA led to significant changes in therapeutic management for $28 \%$ of the patients $(p<0.001)$. nCLE significantly increased the interobserver agreement of $0.28(p<0.0001)$, from 0.36 (CI 95\% 0.33-0.49) to 0.64 (CI 95\% 0.61-0.67). nCLE improved the rates of full agreement among the five experts of $24 \%(p<0.0001)$, from 30 to $54 \%$. With nCLE, the surveillance rate of benign SCAs fell by 35\%, from 40 (28/70) to 5\% (4/76).

Conclusion The addition of nCLE to EUS-FNA significantly improves reliability of PCL diagnosis and could impact the therapeutic management of patients with single PCLs. ClinicalTrials.gov number, NCT01563133.
\end{abstract}

Keywords EUS-FNA $\cdot$ Pancreatic cysts $\cdot$ Needle-based confocal laser endomicroscopy

Electronic supplementary material The online version of this article (https://doi.org/10.1007/s00464-019-07062-9) contains supplementary material, which is available to authorized users.

Bertrand Napoléon

bertrand.napoleon@dartybox.com

Hôpital Beaujon, Clichy, France

2 Hôpital Privé Jean Mermoz, Ramsay Générale de Santé, 4 rue Jacqueline Auriol, 69008 Lyon, France

3 Cliniques Universitaires, Saint-Luc, Belgium

4 Centre Hospitalier Universitaire de l'Archet 2, Nice, France

5 Biomnis, Lyon, France

6 Institut Paoli Calmettes, Marseille, France

7 Clinique du Trocadéro, Paris, France

8 Hôpital Rangueil, Toulouse, France

9 Capionis, Bordeaux, France

\begin{tabular}{ll}
\multicolumn{2}{l}{ Abbreviations } \\
BD-IPMN & $\begin{array}{l}\text { Branch duct-intraductal papillary mucinous } \\
\text { neoplasm }\end{array}$ \\
CT & Computed tomography \\
CEA & Carcinoembryonic antigen \\
EUS-FNA & Endoscopic ultrasound fine-needle aspiration \\
IML & Indeterminate mucinous lesion \\
MCN & Mucinous cystic neoplasm \\
MRI & Magnetic resonance imaging \\
nCLE & Needle-based confocal laser endomicroscopy \\
NEN & Neuroendocrine neoplasm \\
PC & Pseudocyst \\
PCLs & Pancreatic cystic lesions \\
SCA & Serous cystadenoma
\end{tabular}

The therapeutic management of pancreatic cystic lesions (PCL) depends on the cyst type. Surveillance or surgical 
resection is considered for premalignant lesions [mucinous cystic neoplasm (MCN), branch-duct intraductal papillary mucinous neoplasm (BD-IPMN), and neuroendocrine neoplasm (NEN)], while the absence of any surveillance is usually proposed for benign cysts [serous cystadenoma (SCA), pseudocyst (PC)] [1]. When the diagnosis of a single PCL larger than $2 \mathrm{~cm}$ remains uncertain after conventional imaging, endoscopic ultrasound fine-needle aspiration (EUS-FNA) with cyst fluid analysis is proposed. Despite the quality of the information provided by EUS, it is not possible to establish the nature of cysts in up to $30 \%$ of cases [2]. FNA cytohistology is inconclusive in more than 50\% of the cases [3], and the carcinoembryonic antigen (CEA) level lacks specificity [4]. The overall diagnostic accuracy remains low at $61 \%$ for both EUS and EUS-FNA [2]. The selection of the most appropriate treatment regimen relies on an accurate characterization of cysts and, therefore, remains suboptimal [5-8]. The consequences of inappropriate treatment for patients in terms of mortality and morbidity are non-negligible with the current standard of care [9-11].

Needle-based confocal laser endomicroscopy (nCLE) is a new imaging modality allowing in vivo real-time imaging at a microscopic level of the inner wall of pancreatic cyst during an EUS-FNA procedure. nCLE feasibility and safety for the assessment of PCLs were demonstrated in a pilot study [12] and were validated in an international multicenter study [13]. Subsequent clinical investigations described the correlation between nCLE images and histological features, and comprehensive nCLE criteria were established for the characterization of the most frequent types of PCL (NEN, MCN, BD-IPMN, SCA, and PC) [14-16]. A recent international study confirmed excellent interobserver and intraobserver agreement for the interpretation of nCLE images for the diagnosis of PCLs [17]. The CONTACT2 clinical trial prospectively validated the very high nCLE sensitivity and specificity for the diagnosis of PCL on a large multicentric series of 78 cases with definitive diagnoses (surgery or cytohistology) [18]. It demonstrated that nCLE diagnostic performance significantly surpassed that of EUS and CEA titration for differentiating mucinous from nonmucinous lesions and benign from premalignant PCLs [18]. Nevertheless, no study has yet evaluated the impact of nCLE on therapeutic management in clinical practice in patients with PCLs. This second phase of the CONTACT2 study aimed to evaluate nCLE impact on therapeutic management through a retrospective analysis of the 206 prospectively included patients with a single PCL.

\section{Materials and methods}

\section{Study population}

The population has been described in the first phase of the CONTACT2 trial published by Napoleon et al. [18]. The inclusion criteria were as follows: diagnosis of large $(\geq 20 \mathrm{~mm}$ ) single PCL identified with CT and/or MRI with at least one cavity larger than $13 \mathrm{~mm}$ (to allow for CEA and cytohistopathological analyses) without evidence of communication with the main pancreatic duct, without chronic calcifying pancreatitis; no evidence of criteria for malignancy (cyst containing solid masses or mural nodules, presence of metastatic nodes, presence of distant metastases, ascites and vascular infiltration); and scheduled for a diagnostic EUS-FNA procedure. The CONTACT study protocol was approved by the Institutional Review Board of the Institut Paoli Calmettes (Marseille, France), by the French Health Authority (Agence Française de Sécurité Sanitaire des Produits de Santé) and was registered on ClinicalTrials.gov with the following identifier: NCT01563133. The study was performed in accordance with Good Clinical Practice guidelines. All the co-authors had access to the study data and reviewed and approved the final manuscript.

\section{Consensus on therapeutic management}

Five pancreatic disease experts (three endosonographers with EUS-FNA expertise and two pancreato-biliary surgeons) from four tertiary hospitals were involved. They were independent from the CONTACT2 prospective study. A consensus to standardize the therapeutic management of patients with PCLs according to the diagnosis and its confidence level was first defined by the panel of experts as follows:

(1) Eight diagnostic options were retained: SCA, MCN, BD-IPMN, PC, NEN, indeterminate mucinous lesion, indeterminate lesion, and other cyst types (including cystic solid pseudopapillary neoplasm, congenital pancreatic cyst, and cystic lymphoma).

(2) Three therapeutic management options were retained: "neither surgery nor surveillance", "surveillance", or "surgery". If the diagnosis was certain, "neither surgery nor surveillance" was recommended for SCAs and PCs; surgery was recommended for MCNs and NENs; either surgery or surveillance was recommended for BD-IPMNs depending on worrisome features observed in EUS; and either surgery or surveillance was recommended for indeterminate mucinous lesions and for 
indeterminate lesions. The therapeutic management of other rare types of cysts relied on cyst aetiology. Confidence level modified therapeutic management. For example, surveillance instead of "neither surveillance nor surgery" was recommended for SCAs associated with fair confidence level.

\section{Expert training}

The five independent pancreatic experts were informed about nCLE diagnostic performance by two nCLE experts (B.N and M.P) through teleconference. A slide deck was delivered summarizing the comprehensive diagnostic performance of nCLE criteria previously published for the most frequent types of PCLs (NEN, BD-IPMN, MCN, SCA, and PC) $[18,19]$. Figure 1 provides illustrations of the common patterns of nCLE in PCLs. Prospectively validated nCLE diagnostic performances were presented, highlighting the very high specificity of nCLE for the diagnosis of SCA, IPMN, MCN and for the differentiation of mucinous from non-mucinous lesions $[18,19]$. The five experts were also informed about nCLE limitations: first, the nCLE criterion "field of bright, gray or black particle" lacked specificity for PC characterization. It can be observed in other types of cysts such as the inflammatory cells that can be found in the cystic fluid of cystic tumors following infection, bleeding or previous procedures involving punctures; second, the nCLE criterion "dark spots of cell aggregates surrounded by gray areas of fibrosis and vessels" lacks specificity for NENs. It can be observed in other types of premalignant PCLs; third, the MCN nCLE criterion "epithelial borders" and the BD-IPMN nCLE criterion "papillae", can be both observed in the same cyst, leading to the nCLE diagnosis of indeterminate mucinous lesions.

\section{Expert evaluations (Fig. 2)}

The panel of experts independently reviewed every case blinded to one another's decisions, to the final diagnoses and to patient therapeutic managements. Two evaluations were performed. The information for each patient was sequentially disclosed in a stepwise manner: progressively from clinical data, to EUS report and to FNA reports comprising histocytological analysis, CEA and amylase levels. nCLE
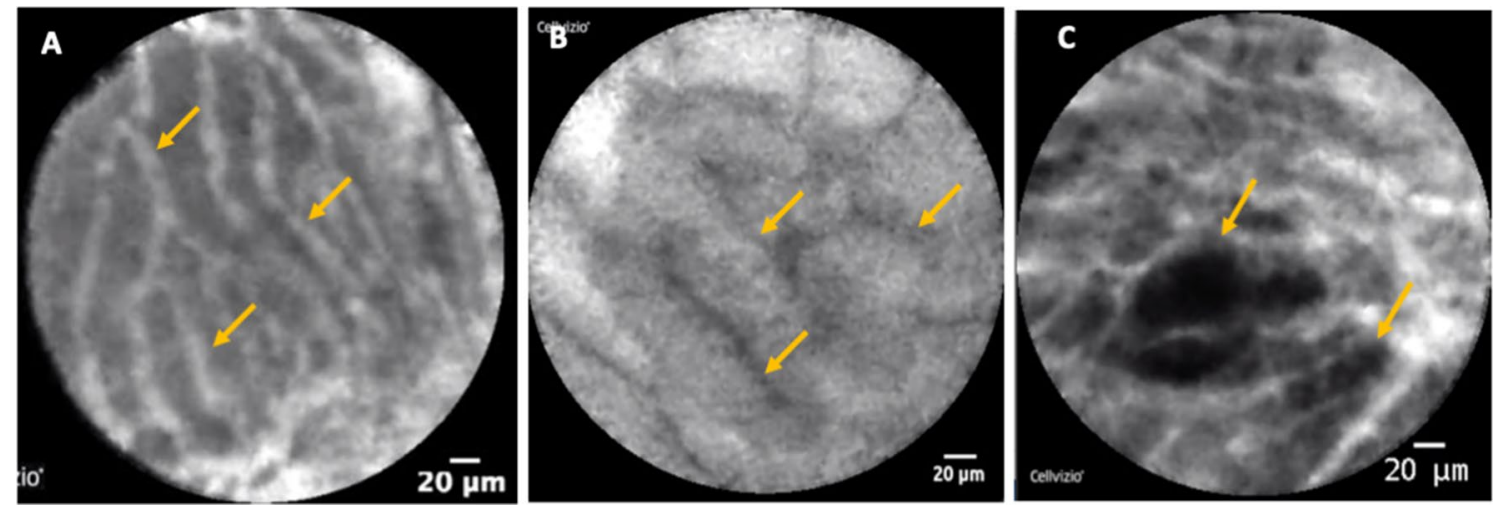

Fig. 1 Common patterns of nCLE in pancreatic cystic tumors. A Serous cystadenoma: superficial vascular network in white (arrow) filled with fluorescein; black particles inside the vessels correspond to red cells. B IPMN: multiple papillae (arrows) with epithelial border in dark gray. $\mathbf{C}$ cystic $N E N$ irregular clusters of tumoral cells (arrows)

Evaluation 1

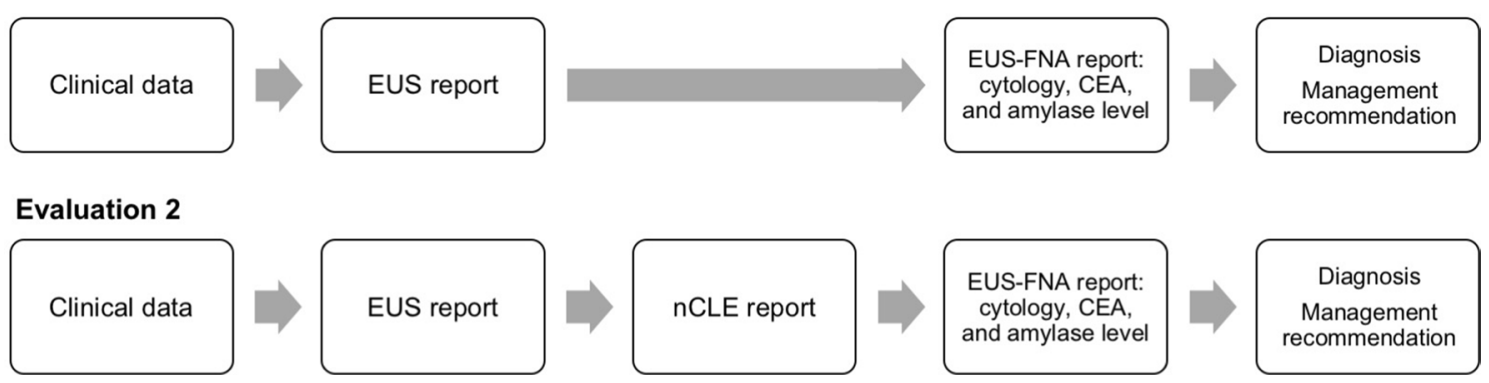

Fig. 2 Flowcharts of evaluation 1 (EUS-FNA) and evaluation 2 (EUS-nCLE-FNA). EUS-FNA endoscopic ultrasound-guided fine-needle aspiration, $n C L E$ needle-based confocal laser endomicroscopy, $C E A$ carcinoembryogenic antigen 
data were not available in evaluation 1, referred to as "EUSFNA" (the current standard of care for diagnosis). In evaluation 2, referred as "EUS-nCLE-FNA", the nCLE report, describing prospectively observed nCLE criteria, their associated diagnoses and the quality of nCLE images, was given before the FNA reports. Patients were randomly reordered between evaluations 1 and 2. Evaluation 2 was performed at least 15 days after evaluation 1 . For each patient and each evaluation, each expert had to retain one of the eight diagnosis and one of the three therapeutic management options.

\section{Statistical analyses}

Baseline characteristics, including demographic and clinical data, were described as percentages and ranges or means and standard deviations, as appropriate. Diagnostic yields were defined as the ratio of conclusive tests (nCLE and FNA cytohistology) over the total number of patients.

To assess interobserver agreement (IOA) between the five experts, Fleiss' kappas were calculated for the proposed diagnoses and therapeutic management for evaluations 1 and 2. The R package "raters" was used to determine Fleiss' kappa with 95\% CI. Fleiss' Kappa was interpreted using Landis and Koch-Kappa's benchmark scale.

For each patient, final consensus on diagnosis and therapeutic management were determined with a majority of at least $3 / 5$ of the experts. The absence of a final consensus led to indeterminate answers. A consensus on the diagnosis of indeterminate mucinous lesions was determined when a majority of experts proposed a diagnosis belonging to the overall group of mucinous lesions (IPMN, MCN, indeterminate mucinous lesion) in the absence of a majority for a more specific diagnosis (IPMN, MCN). The impact on diagnosis was defined as the change in the final consensus diagnosis between evaluations 1 and 2 . The impact on therapeutic management was defined as the change in the final therapeutic management consensus between evaluations 1 and 2 .

Overall diagnostic and therapeutic management proportion changes between evaluations 1 and 2 were compared using the $\chi^{2}$ square test. Changes between evaluations 1 and 2 regarding the specific diagnostic and therapeutic management categories were compared using McNemar's test. Fleiss' kappas from evaluations 1 and 2 were compared using the $Z$ test. $p<0.05$ was considered statistically significant.

\section{Endpoints}

The main objective of the study was to evaluate the impact of nCLE on PCL therapeutic management: a significant proportion of final consensus changes between evaluations 1 and 2 for a given therapeutic management option was defined as the primary endpoint. The secondary objectives of the study were to evaluate the impact of nCLE on diagnosis and on IOAs for both diagnosis and therapeutic management: a significant proportion of final consensus changes for a given type of diagnosis, a significant increase of IOAs and an increased rate of full agreement between the five experts, between evaluations 1 and 2, for both diagnosis and therapeutic management, were also defined as secondary endpoints.

\section{Results}

\section{Demographics and clinical data}

A prospective database of patients with large single PCL examined by EUS-FNA and nCLE was derived from the CONTACT2 study (Supplementary Fig. 1). In this trial, 217 patients with PCLs were potentially eligible. Eight were excluded, leaving 209 eligible patients. With two failures of the puncture procedure and 1 nCLE imaging failure, EUS-nCLE-FNA procedures were successfully performed in $99 \%$ of the cases (206/209). The demographic and clinical data of the 206 analyzable patients are described in Tables 1 and 2. Overall FNA cytohistology was contributory in $30 \%$ of the cases (61/206). A conclusive nCLE test (identified nCLE criteria) was obtained in 175 patients, leading to an overall nCLE diagnostic yield of $85 \%$ (175/206). CEA was available in $76 \%$ of the cases $(157 / 206)$, with a level $>192 \mathrm{ng} / \mathrm{mL}$ in $35 \%(55 / 157)$ of the cases and $<5 \mathrm{ng} / \mathrm{mL}$ in $38 \%(59 / 157)$ of the cases. Three cases of post-procedure pancreatitis (1.4\%) were reported. No adverse events associated with fluorescein injection were noted.

\section{Impact of $\mathbf{n C L E}$ on PCL diagnoses}

The addition of nCLE information to standard EUS-FNA analysis significantly changed the proposed diagnoses in $27 \%$ of the cases $(57 / 206)(p=0.005)$ (Supplementary Table 1). Combined EUS-nCLE-FNA allowed a significant increase of BD-IPMNs diagnoses, from 48 to $65(p=0.002)$, significantly decreasing IML diagnoses from 35 to 16 $(p=0.001)$ (Fig. 3). Indeed, EUS-nCLE-FNA refined 23 indeterminate mucinous lesions into $16 \mathrm{BD}-\mathrm{IPMNs}$ and 7 MCNs (Supplementary Table S1). The number of SCA diagnoses also significantly increased from 70 to $76(p=0.034)$ (Supplementary Table S1). The number of indeterminate cysts numerically but not significantly decreased from 18 to 11 ( $p=0.108)$ (Fig. 3). The addition of nCLE did not significantly impact the overall numbers of proposed NEN, MCN, PC, and other diagnoses (Fig. 3). 
Table 1 Demographic and clinical data for all cysts

\begin{tabular}{|c|c|}
\hline \multicolumn{2}{|l|}{ Patients $(n=206)$} \\
\hline Age, mean (range), years & $57(23-84)$ \\
\hline Male $[n(\%)]$ & $69(33)$ \\
\hline \multicolumn{2}{|l|}{ Pre-existing conditions $[n(\%)]$} \\
\hline Previous EUS-FNA & $64(33)$ \\
\hline \multicolumn{2}{|l|}{ Symptoms $[n(\%)]$} \\
\hline No symptoms & $154(75)$ \\
\hline Acute pancreatitis & $16(8)$ \\
\hline Aspecific abdominal pain & $36(17)$ \\
\hline \multicolumn{2}{|l|}{ Cyst morphology } \\
\hline \multicolumn{2}{|l|}{ Location $[n(\%)]$} \\
\hline Uncinate & $10(49)$ \\
\hline Head & $58(28)$ \\
\hline Neck & $27(13)$ \\
\hline Body & $67(33)$ \\
\hline Tail & $44(21)$ \\
\hline Lesion size, mean (range), $\mathrm{mm}$ & $38(20-200)$ \\
\hline Main pancreatic duct dilation $[n(\%)]$ & $19(9)$ \\
\hline \multicolumn{2}{|l|}{ Number of cavities $[n(\%)]$} \\
\hline 1 & $88(43)$ \\
\hline 2 & $22(11)$ \\
\hline$>2$ and $<10$ & $58(28)$ \\
\hline$\geq 10$ & $38(18)$ \\
\hline Wall thickness $\geq 1 \mathrm{~mm}[n(\%)]$ & $32(16)$ \\
\hline Presence of calcification $[n(\%)]$ & $18(9)$ \\
\hline \multicolumn{2}{|l|}{ Intracystic concentrations } \\
\hline $\mathrm{CEA}>192 \mathrm{ng} / \mathrm{mL}[n(\%)]$ & $55(27)$ \\
\hline $\mathrm{CEA}<5 \mathrm{ng} / \mathrm{mL}[n(\%)]$ & $59(29)$ \\
\hline Amylase <250 IU/L [ $n(\%)]$ & $63(31)$ \\
\hline
\end{tabular}

$C E A$ carcinoembryogenic antigen, EUS-FNA endoscopic ultrasoundguided fine-needle aspiration

\section{Impact of $\mathrm{nCLE}$ on therapeutic management of patients with PCLs}

The addition of nCLE to the standard EUS-FNA procedure significantly changed $28 \%$ (58/206) of the overall proposed therapeutic managements. nCLE significantly increased the number of "neither surgery nor surveillance" recommendations, from 45 to $79(p<0.0001)$ and decreased surveillance recommendations, from 122 to $88(p<0.0001)$ (Fig. 4). The addition of nCLE to EUS-FNA analysis changed the therapeutic management for 33 patients from surveillance to "neither surgery nor surveillance" (Supplementary Table S2). Thirty-two of those 33 patients were diagnosed with SCAs by EUS-nCLE-FNA. The overall number of proposed surgeries was not statistically impacted by nCLE (Fig. 4). However, 6 of the 34 patients (18\%) who were recommended for surgery in evaluation 1 were reconsidered for surveillance after the addition of nCLE information in evaluation 2 , while 9 of 122 patients (7\%) in the surveillance group in evaluation
Table 2 Technical feasibility and safety for all cysts

\begin{tabular}{|c|c|}
\hline \multicolumn{2}{|l|}{ Cyst access $[n(\%)]$} \\
\hline Easy & $188(91)$ \\
\hline Moderate or difficult & $18(9)$ \\
\hline \multicolumn{2}{|l|}{ Needle type $[n(\%)]$} \\
\hline 19 G Echo Tip Ultra* & $21(10)$ \\
\hline 19 G Expect Flexible ${ }^{\dagger}$ & $185(90)$ \\
\hline \multicolumn{2}{|l|}{ Access route $[n(\%)]$} \\
\hline Transgastric & $140(68)$ \\
\hline Transduodenal & $66(32)$ \\
\hline Second part of the duodenum & $5(2)$ \\
\hline \multicolumn{2}{|l|}{ Miniprobe extraction from the needle $[n(\%)]$} \\
\hline Possible & $195(95)$ \\
\hline Not possible (extracted together with the needle) & $11(5)$ \\
\hline \multicolumn{2}{|l|}{ Cytohistology $[n(\%)]$} \\
\hline Contributive & $61(30)$ \\
\hline Non-contributive & $145(70)$ \\
\hline \multicolumn{2}{|l|}{ Biochemical dosage $[n(\%)]$} \\
\hline Successful & $157(76)$ \\
\hline Unsuccessful & $49(24)$ \\
\hline Insufficient fluid volume & $34(17)$ \\
\hline Intracystic bleeding (hematic fluid) & $8(4)$ \\
\hline High viscosity & $4(1.9)$ \\
\hline Fluid leak & $3(1.4)$ \\
\hline \multicolumn{2}{|l|}{ Safety $[n(\%)]$} \\
\hline Post-procedure pancreatitis & $3(1.4)$ \\
\hline
\end{tabular}

1 were recommended for surgery in evaluation 2 (Supplementary Table 2). Those nine patients were diagnosed with mucinous lesions by EUS-nCLE-FNA.

\section{Impact of nCLE on interobserver agreements}

Regarding proposed diagnoses, EUS-nCLE-FNA Fleiss' kappa was substantial $(0.70$, CI 95\% 0.67-0.72) in the overall population of cysts and was significantly increased by 0.25 compared to that of EUS-FNA (0.45, CI 95\% 0.43-0.47) $(p<0.0001)$. nCLE increased the rate of full agreement on diagnosis among the five experts from 29 (59/206) to $61 \%$ $(125 / 206)(p<0.0001)$ (Fig. 5A). For proposed therapeutic management, EUS-nCLE-FNA Fleiss' kappa was substantial $(0.64$, CI 95\% 0.61-0.67) in the overall population of cysts and was significantly increased by 0.28 compared to that of EUS-FNA $(0.36$, CI 95\% 0.33-0.49) $(p<0.0001)$. nCLE increased the rate of full agreement on therapeutic management among the five experts from $30 \%(62 / 206)$ to $54 \%(112 / 206)(p<0.0001)$ (Fig. 5B). 

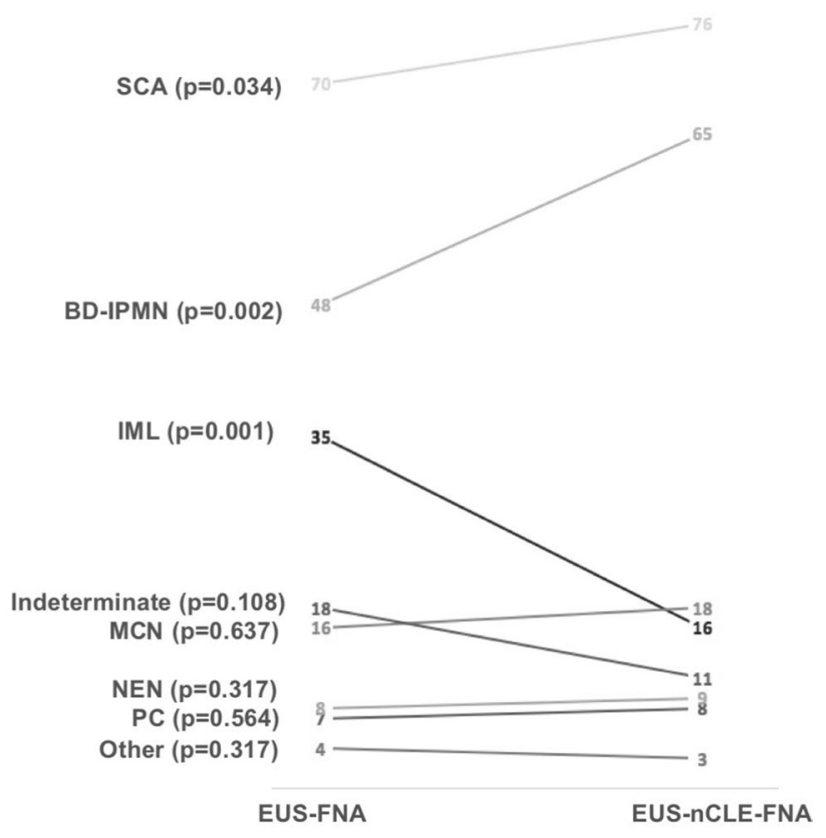

Fig. 3 Diagnoses consensus in evaluations 1 (EUS-FNA) and 2 (EUS-nCLE-FNA). $B D-I P M N$ branch duct-intraductal papillary mucinous neoplasm, $I M L$ indeterminate mucinous lesion, $M C N$ mucinous cystadenoma, SCA serous cystadenoma, $P C$ pseudocyst, $N E N$ neuroendocrine neoplasm, EUS endoscopic ultrasonography, FNA fine-needle aspiration, $n C L E$ needle-based confocal laser endomicroscopy. $p$ values from McNemar test are indicated for each diagnostic option

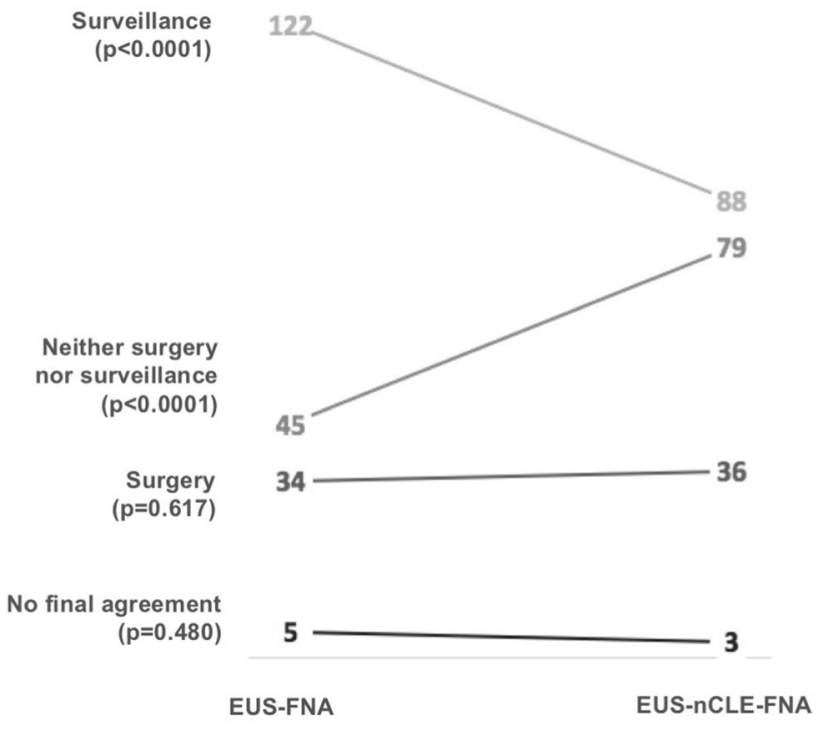

Fig. 4 Therapeutic management according to evaluations 1 (EUSFNA) and 2 EUS-nCLE-FNA). EUS endoscopic ultrasonography, $F N A$ fine-needle aspiration, $n C L E$ needle-based confocal laser endomicroscopy. $p$ values from McNemar test are indicated for each diagnostic option
All authors had access to the study data and reviewed and approved the final version of the manuscript.

\section{Discussion}

Increasing numbers of incidental PCLs have been identified due to increasing use of computed tomography (CT) scans and magnetic resonance imaging (MRI) [20, 21]. Single noncommunicating PCL without chronic calcified pancreatitis represents the most challenging diagnostic issue and should be carefully considered because of the malignant potential of some types of PCLs. In current practice, the therapeutic management of PCLs usually relies on multidisciplinary review board decisions based on cyst imaging characteristics, CEA levels and cytohistopathological analyses. Using this approach for 206 patients extracted from a prospective database, the IOA Fleiss' kappas between a panel of experts in pancreatic diseases were dramatically low for both PCL diagnosis $(0.45)$ and therapeutic management $(0.36)$. These results can be explained by the low rate of $30 \%$ of contributory FNA cytohistopathological analyses and by the lack of accuracy of CEA level. These data are consistent with those of the literature [22] and emphasize the necessity of a more reliable diagnostic method for the diagnosis of PCLs.

In our series, the overall diagnostic yield of nCLE (85\%) was significantly higher than that of FNA cytohistopathology (30\%). The addition of nCLE to EUS-FNA allowed significant changes of diagnoses and therapeutic managements in $27 \%$ and $28 \%$ of the cases, respectively (with $p=0.005$ and $p<0.0001$, respectively), while IOA Fleiss' kappas among the experts significantly increased from 0.45 to 0.70 for diagnosis and from 0.36 to 0.64 for therapeutic management strategies. Strikingly, nCLE increased the rate of full agreement among the panel of experts from 29 to $61 \%$ for diagnoses and from 30 to $54 \%$ for therapeutic managements. This effect of nCLE increased the value of multidisciplinary review boards with less disagreement among participants.

The addition of nCLE significantly increased the number of BD-IPMNs diagnoses $(p=0.002)$ at the expense of a significant decrease of IML $(p=0.001)$. Indeed, EUS-nCLEFNA refined 23 indeterminate mucinous lesions into 16 BD-IPMNs and 7 MCNs. These data emphasize that nCLE is useful to ascertain the diagnosis of BD-IPMN and should be recommended for a more specific diagnosis. nCLE also increased the number of SCA diagnoses $(p=0.034)$ and the number of "neither surgery nor surveillance" recommendations $(p<0.0001)$ while decreasing surveillance recommendations $(p<0.0001)$; the surveillance rate of SCAs fell from $40(28 / 70)$ to $5 \%(4 / 76)$. These data show that nCLE is essential to ascertain the diagnosis and the therapeutic management of SCA by allowing the identification of its specific "superficial vascular network". nCLE should be at 
Fig. 5 Agreement between the panel of five experts. A Agreement among the eight possible diagnosis options (NEN, BD-IPMN, MCN, indeterminate mucinous lesion, SCA, PC, indeterminate or other) according to evaluation 1 (EUS-FNA) and evaluation 2 (EUS-nCLEFNA). B Agreement among the three possible therapeutic management options ("neither surgery nor surveillance", "surveillance" or "surgery") according to evaluation 1 and evaluation 2. EUS endoscopic ultrasonography, FNA fineneedle aspiration, $n C L E$ needlebased confocal laser endomicroscopy, $k$ Fleiss' kappa. NEN neuroendocrine neoplasm, $B D$ $I P M N$ branch duct-intraductal papillary mucinous neoplasm, $M C N$ mucinous cystadenoma, $S C A$ serous cystadenoma, $P C$ pseudocyst
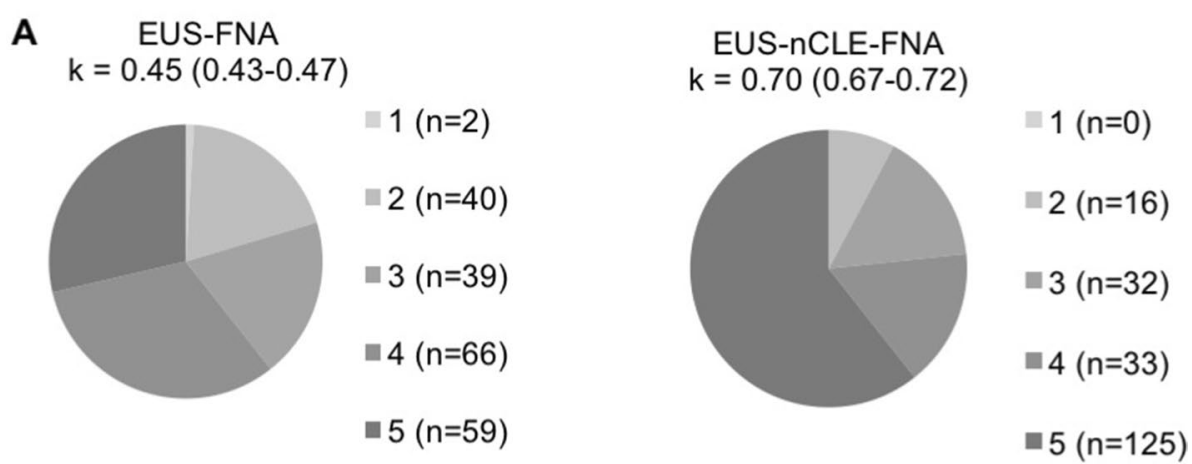

B

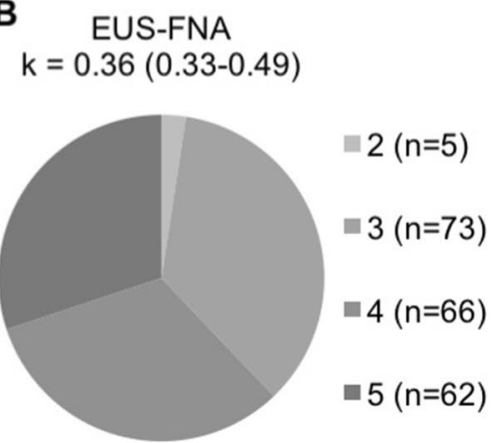

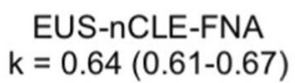

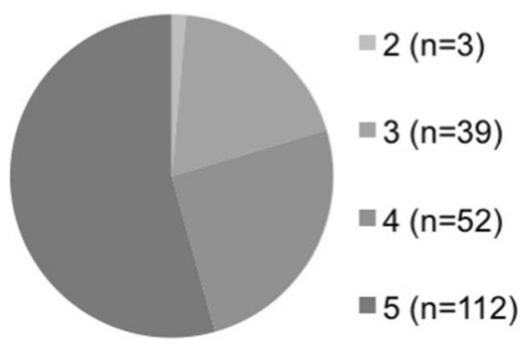

least recommended to ascertain the presumptive diagnosis of SCA. Moreover, a recent French health economic model reported that nCLE may be cost-effective for the therapeutic management of SCA [23]. In this medico-economic analysis, the additional cost per patient for nCLE was estimated at $600 €$. This analysis showed that the addition of nCLE to EUS-FNA resulted in a reduction of $23 \%$ in the total rate of surgical intervention, which translated to a reduction in clinical costs of $13 \%$ (public sector) and 14\% (private sector). Therefore, nCLE should be systematically considered when EUS-FNA is indicated for the evaluation of PCLs."

Our data confirmed the safety of nCLE in the largest multicentric cohort of 206 patients who underwent EUS$\mathrm{FNA}+\mathrm{nCLE}$ procedures. Indeed, the rate of post-procedure acute pancreatitis in the trial was $1.4 \%$ (3/206). This incidence is similar to the previously reported risk levels following standard EUS-FNA procedures [24] and is lower than those of nCLE pilot studies [12]. The necessity of using a 19G needle to perform nCLE procedure represents the main technical limitation. Two technical failures of cyst puncture were observed in lesions only accessible from the second part of the duodenum, which is consistent with previously reported experience [15].

Our study had several limitations: (1) only a few of the PCLs included in the study had a surgical gold standardbased diagnosis. This is inherent to the therapeutic management of the studied disease, especially when dealing with presumptive benign PCLs for which ethical considerations obviously prevent systematic resections. For the other PCLs, the follow-up was not very long term. Therefore, the diagnoses proposed by the experts are tentative. Thus, the only certain finding is that the use of nCLE increases interobserver agreement between specialists. The comparison of retrospective data analyses with a prospectively obtained gold standard diagnosis would have introduced a major methodological bias. Nevertheless, two large prospective studies have already validated the very high diagnostic performance of nCLE for all types of cysts $[17,18]$ and have demonstrated the almost perfect interobserver reliability of nCLE criteria [17]. (2) The contribution of CT and MRI outcomes was not evaluated in our study due to the heterogeneity of data collection. Nevertheless, this would probably not have affected the results because the patients included in our cohort were sent for EUS-FNA after inconclusive CT and MRI. (3) We did not include in our study new PCLs diagnostic tools for which the potential interests have been published during the conduct of the study, including DNA mutations [25, 26], string sign tests [27], and EUS-guided microforceps biopsies [28]. (4) For the sake of simplification, therapeutic management was based on a consensus of a panel of five experts. Systematic resection was recommended for all patients diagnosed with MCN, whereas surveillance was recommended for BD-IPMN without worrisome features [1]. This can be discussed as the most recent guidelines propose observation as an option for asymptomatic MCNs $<4 \mathrm{~cm}$ without mural nodules [22, 29]. Nevertheless, MCNs are predominantly present in the body or tail of the pancreas of middle-age women and are definitely cured without the need 
for follow-up in the absence of cancer. The natural history of MCN remains unknown and non-operative management would require years of follow-up based on high-resolution imaging associated with high costs [1]. Therefore, in most centers, resection remains proposed for $\mathrm{MCN}$.

\section{Conclusion}

The diagnosis of large single pancreatic cysts represents a major issue for clinicians. The addition of nCLE information to EUS-FNA significantly improves the reliability both in diagnosis and therapeutic management among experts in pancreatic cystic lesions. These results support the recognition of nCLE as a key tool of the standard of care for such clinical situations.

Acknowledgements We would like to thank all the procedural nurses and staff for their assistance during procedures. We would like to thank Leslie Assouline and Guillaume Trébuchet from Mauna Kea Technologies for additional logistical support during the trial.

Author contributions Conception or design of the work (Dr. M. Palazzo, Dr. B. Napoleon, Dr. S. Marque); data collection (all authors); data analysis and interpretation (Dr. B. Napoleon, Dr. AI. Lemaistre, Dr. M. Morellon); drafting the article (Dr. B. Napoleon, Dr. M. Palazzo); critical revision of the article (all authors); final approval of the version to be published (all authors).

Funding This study has been funded by Mauna Kea Technologies. Mauna Kea Technologies provided logistical support during the trial.

\section{Compliance with ethical standards}

Disclosure Drs. B. Napoléon, Dr M. Palazzo is on the speakers' bureau of Mauna Kea Technologies. Drs. B. Napoléon, M. Palazzo, A. Sauvanet, R. Gincul, I. Borbath, G. Vanbiervliet, R. Bourdariat, A.-I. Lemaistre, B. Pujol, F. Caillol, L. Palazzo, A. Aubert, F. Maire, L. Buscail, and M. Giovannini have received financial support from Mauna Kea Technologies. Dr. B. Napoléon has also received financial support from Cook Medical Corporation, Ipsen, Boston Scientific Corporation, Olympus Corporation and Cousin Corporation. R. Gincul has also received financial support from Ipsen. Dr. G. Vanbiervliet has also received financial support from Cook Medical Corporation and Boston Scientific Corporation. Dr. S. Marque has no conflicts of interest or financial ties to disclose.

Open Access This article is distributed under the terms of the Creative Commons Attribution 4.0 International License (http://creativeco mmons.org/licenses/by/4.0/), which permits unrestricted use, distribution, and reproduction in any medium, provided you give appropriate credit to the original author(s) and the source, provide a link to the Creative Commons license, and indicate if changes were made.

\section{References}

1. Tanaka M, Fernández-del Castillo C, Adsay V et al (2012) International consensus guidelines 2012 for the management of IPMN and MCN of the pancreas. Pancreatology 12:183-197

2. Rodríguez-D’Jesús A, Fernández-Esparrach G, Boadas J et al (2016) Impact of endoscopic ultrasonography (EUS) and EUSguided fine-needle aspiration on the management of pancreatic cystic lesions. Eur J Gastroenterol Hepatol 28:1094-1099

3. Thornton GD, McPhail MJW, Nayagam S et al (2013) Endoscopic ultrasound guided fine needle aspiration for the diagnosis of pancreatic cystic neoplasms: a meta-analysis. Pancreatology 13:48-57

4. Brugge WR, Lewandrowski K, Lee-Lewandrowski E et al (2004) Diagnosis of pancreatic cystic neoplasms: a report of the cooperative pancreatic cyst study. Gastroenterology 126:1330-1336

5. Bhutani MS (2004) Role of endoscopic ultrasonography in the diagnosis and treatment of cystic tumors of the pancreas. JOP J Pancreas 5:266-272

6. van der Waaij LA, van Dullemen HM, Porte RJ (2005) Cyst fluid analysis in the differential diagnosis of pancreatic cystic lesions: a pooled analysis. Gastrointest Endosc 62:383-389

7. Belsley NA, Pitman MB, Lauwers GY et al (2008) Serous cystadenoma of the pancreas: limitations and pitfalls of endoscopic ultrasound-guided fine-needle aspiration biopsy. Cancer 114:102-110

8. Park WG-U, Mascarenhas R, Palaez-Luna M et al (2011) Diagnostic performance of cyst fluid carcinoembryonic antigen and amylase in histologically confirmed pancreatic cysts. Pancreas 40:42-45

9. Jais B, Rebours V, Malleo G et al (2016) Serous cystic neoplasm of the pancreas: a multinational study of 2622 patients under the auspices of the International Association of Pancreatology and European Pancreatic Club (European Study Group on Cystic Tumors of the Pancreas). Gut 65:305-312

10. Gaujoux S, Brennan MF, Gonen M et al (2011) Cystic lesions of the pancreas: changes in the presentation and management of 1,424 patients at a single institution over a 15 -year time period. $\mathbf{J}$ Am Coll Surg 212:590-600

11. Valsangkar NP, Morales-Oyarvide V, Thayer SP et al (2012) 851 resected cystic tumors of the pancreas: a 33-year experience at the Massachusetts General Hospital. Surgery 152:S4-S12

12. Konda VJA, Aslanian HR, Wallace MB et al (2011) First assessment of needle-based confocal laser endomicroscopy during EUS-FNA procedures of the pancreas (with videos). Gastrointest Endosc 74:1049-1060

13. Konda V, Meining A, Jamil L et al (2013) A pilot study of in vivo identification of pancreatic cystic neoplasms with needle-based confocal laser endomicroscopy under endosonographic guidance. Endoscopy 45:1006-1013

14. Napoléon B, Lemaistre A-I, Pujol B et al (2015) A novel approach to the diagnosis of pancreatic serous cystadenoma: needle-based confocal laser endomicroscopy. Endoscopy 47:26-32

15. Napoleon B, Lemaistre A-I, Pujol B et al (2016) In vivo characterization of pancreatic cystic lesions by needle-based confocal laser endomicroscopy (nCLE): proposition of a comprehensive nCLE classification confirmed by an external retrospective evaluation. Surg Endosc 30:2603-2612

16. Krishna SG, Swanson B, Hart PA et al (2016) Validation of diagnostic characteristics of needle based confocal laser endomicroscopy in differentiation of pancreatic cystic lesions. Endosc Int Open 4:E1124-E1135

17. Krishna SG, Brugge WR, Dewitt JM et al (2017) Needlebased confocal laser endomicroscopy for the diagnosis of pancreatic cystic lesions: an international external interobserver 
and intraobserver study (with videos). Gastrointest Endosc 86(4):644-654

18. Napoleon B, Palazzo M, Lemaistre A-I et al (2018) Needle-based confocal laser endomicroscopy of pancreatic cystic lesions: a prospective multicenter validation study in patients with definite diagnosis. Endoscopy. https://doi.org/10.1055/a-0732-5356

19. Napoleon B, Pujol B, Palazzo M et al (2017) Needle-based confocal laser endomicroscopy (nCLE) for the diagnosis of pancreatic cystic lesions: preliminary results of the first prospective multicenter study. Gastroenterology 152:S132-S133

20. Laffan TA, Horton KM, Klein AP et al (2008) Prevalence of unsuspected pancreatic cysts on MDCT. Am J Roentgenol 191:802-807

21. de Jong K, Nio CY, Hermans JJ et al (2010) High prevalence of pancreatic cysts detected by screening magnetic resonance imaging examinations. Clin Gastroenterol Hepatol 8:806-811

22. European Study Group on Cystic Tumours of the Pancreas (2018) European evidence-based guidelines on pancreatic cystic neoplasms. Gut 67:789-804

23. Le Pen C, Palazzo L, Napoléon B (2017) A health economic evaluation of needle-based confocal laser endomicroscopy for the diagnosis of pancreatic cysts. Endosc Int Open 5:E987-E995

24. Zhu H, Jiang F, Zhu J et al (2017) Assessment of morbidity and mortality associated with endoscopic ultrasound-guided fine-needle aspiration for pancreatic cystic lesions: a systematic review and meta-analysis. Dig Endosc Off J Jpn Gastroenterol Endosc Soc 29:667-675

25. Kung JS, Lopez OA, McCoy EE et al (2014) Fluid genetic analyses predict the biological behavior of pancreatic cysts: three-year experience. JOP J Pancreas 15:427-432

26. Springer S, Wang Y, Dal Molin M et al (2015) A combination of molecular markers and clinical features improve the classification of pancreatic cysts. Gastroenterology 149:1501-1510

27. Bick B, Enders F, Levy M et al (2015) The string sign for diagnosis of mucinous pancreatic cysts. Endoscopy 47:626-631

28. Yang D, Trindade AJ, Yachimski P, Benias P, Nieto J, Manvar A, Ho S, Esnakula A, Gamboa A, Sethi A, Gupte A (2019) Histologic analysis of endoscopic ultrasound-guided through the needle microforceps biopsies accurately identifies mucinous pancreas cysts. Clin Gastroenterol Hepatol 17:1587-1596

29. Elta GH, Enestvedt BK, Sauer BG et al (2018) ACG clinical guideline: diagnosis and management of pancreatic cysts. Am J Gastroenterol 113:464-479

Publisher's Note Springer Nature remains neutral with regard to jurisdictional claims in published maps and institutional affiliations. 